\title{
Protective effect of anthocyanin on lung ischemia-reperfusion injury
}

\author{
Yu S., Wenling Z., Jing L., Bin L. \\ Sichuan University, Dept of Anaesthesiology, Chengdu, China
}

\section{Background and Goal of Study}

Cyanidin-3-O-b-glucoside (C3G), a typical anthocyanin pigment that exists in the human diet, has been reported to protect the heart [1], brain [2], liver [3] and other organs [4] from ischemia/reperfusion (I/R)-induced inflammatory and apoptosis. However, unclear is the potential effect of $\mathrm{C} 3 \mathrm{G}$ on Lung ischemia-reperfusion injury (LIRI). Thus, we hypothesized that $\mathrm{C} 3 \mathrm{G}$ treatment plays a vital role in relieving LIRI.

\section{Materials and Methods}

Forty eight C57BL/6J adult male wild-type mice ( $\mathrm{n}=12$ /group, 20$25 \mathrm{~g})$, were randomly and evenly divided to $\mathrm{I} / \mathrm{R}+\mathrm{C} 3 \mathrm{G}(\mathrm{I} / \mathrm{R}+\mathrm{C})$ group, $\mathrm{I} / \mathrm{R}+$ saline $(\mathrm{I} / \mathrm{R})$ group, sham $+\mathrm{C} 3 \mathrm{G}(\mathrm{sham}+\mathrm{C})$ group and sham+saline (sham) group. LIRI mouse model was built via one-hour occlusion of the left pulmonary hilum and two-hour reperfusion. C3G(40mg/kg) and same dose of $0.9 \%$ sterile saline were administered to $\mathrm{I} / \mathrm{R}+\mathrm{C}$ group and other groups in advance, respectively. Blood and lung tissues of all mice were harvested after lung $\mathrm{I} / \mathrm{R}$ for subsequent analysis. Arterial blood gas (ABG) analysis, Lung Wet/dry (w/d) weight ratio analysis, and Histological analysis were performed. The levels of inflammatory cytokines, including tumor necrosis factor- $\alpha$ (TNF- $\alpha$ ), interleukin-6 (IL-6) and nuclear factor- $\mathrm{kB}$ p65 (NF- $\mathrm{kB}$ p65) activation in the serum and lung tissue, were measured by ELISA, Western blot (WB) and Quantitative polymerase chain reaction (qPCR), respectively. Also, toll-like receptor 3 (TLR3) levels were measured by the same methods. The apoptosis assays, including cleaved caspase- 3 and TUNEL, were assessed.

Results and Discussion: The results exhibited significantly increased lung injury scores based on the outcomes of $A B G$, w/d weight ratio and lung Histological analysis after I/R. Moreover, the results of ELISA, WB and PCR showed the cytokine production including TNF- $\alpha$, IL- 6 and NF- $\mathrm{KB} 65$ activation, and TLR3 levels were substantially raised in $\mathrm{I} / \mathrm{R}$ group. Pulmonary apoptosis after $\mathrm{I} / \mathrm{R}$ was elevated as shown in TUNEL staining and activated caspase- 3 . C $3 \mathrm{G}$ treatment, in contrast, markedly reduced the and pulmonary apoptosis in $\mathrm{I} / \mathrm{R}+\mathrm{C}$ group. In addition, pulmonary and circulating TLR 3 levels in $\mathrm{I} / \mathrm{R}+\mathrm{C}$ group decreased compared with $\mathrm{I} / \mathrm{R}$ group. But the expressions of the inflammatory cytokines in both lung tissue and serum did not show a difference.

Conflicts of interest: The authors have no conflicts of interest

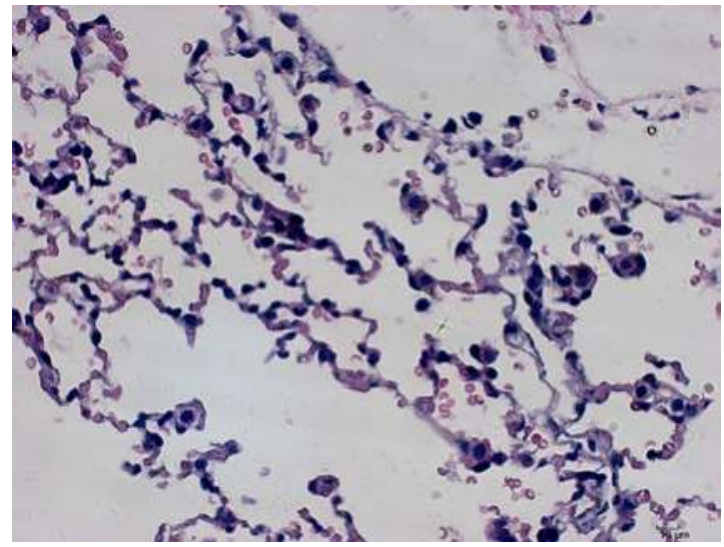

Sham

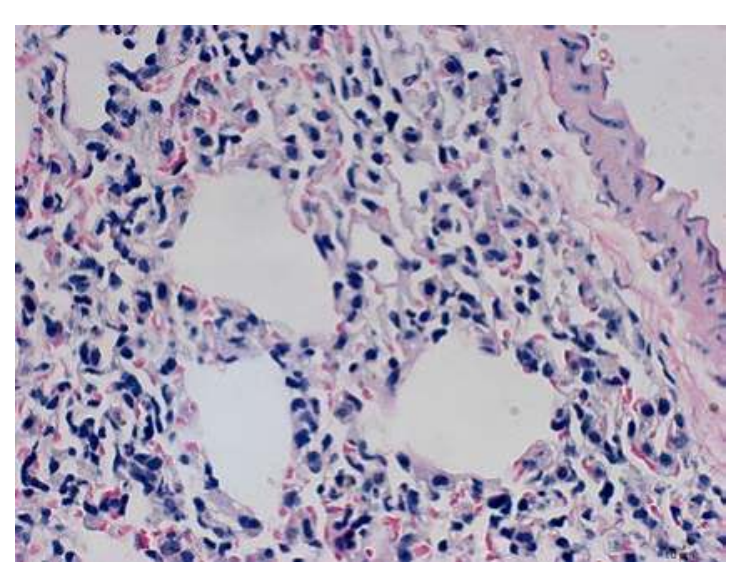

$\mathrm{I} / \mathrm{R}$

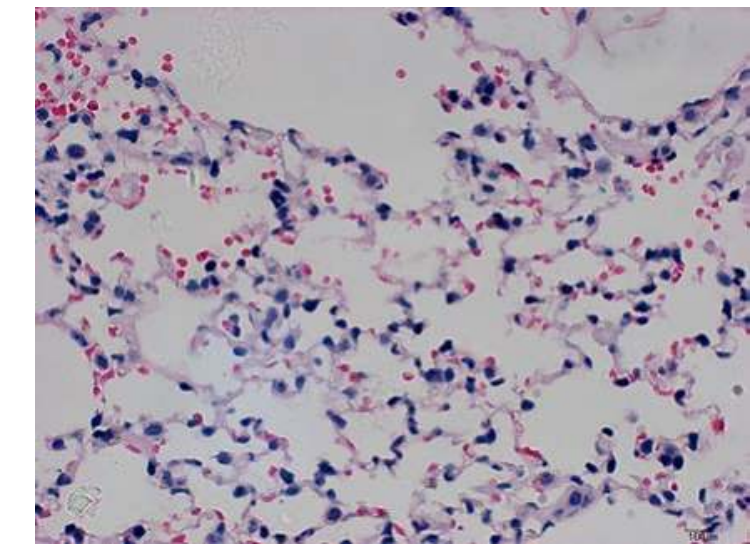

Sham $+\mathrm{C}$

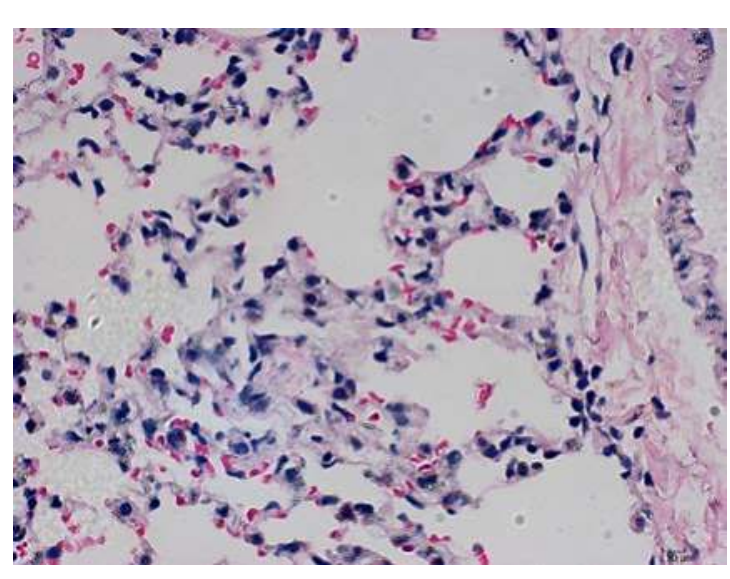

$\mathrm{I} / \mathrm{R}+\mathrm{C}$
Firgure1. Effect of $\mathrm{C} 3 \mathrm{G}$ on lung ischemia/reperfusion injury. Left lung tissues were stained with HE. Representative photomicrographs are at 400 magnification.

Conclusion(s): Injection of $\mathrm{C} 3 \mathrm{G}$ during preoperative period relieved pulmonary apoptosis induced by LIRI. The potential mechanism may be related to the decrease of TLR3 level and the inhibition of inflammation and apoptosis.

\section{References}

1.Amorini, A.M., et al., Cyanidin-3-O - $\beta$-glucopyranoside Protects Myocardium and Erythrocytes from Oxygen Radical-mediated Damages. Free Radical Research, 2009. 37(4): p. 453-460.

2.Shin, W.-H., S.-J. Park, and E.-J. Kim, Protective effect of anthocyanins in middle cerebral artery occlusion and reperfusion model of cerebral ischemia in rats. Life Sciences, 2006. 79(2): p. 130137.

3.Tsuda, T., et al., Cyanidin 3-O-beta-D-glucoside attenuates the hepatic ischemia-reperfusion injury through a decrease in the neutrophil chemoattractant production in rats. J Nutr Sci Vitaminol (Tokyo), 2002. 48(2): p. 134-41.

3.Kim, H.J., et al., Anti-inflammatory effects of anthocyanins from black soybean seed coat on the keratinocytes and ischemia-reperfusion injury in rat skin flaps. Microsurgery, 2012. 32(7): p. 563-70.

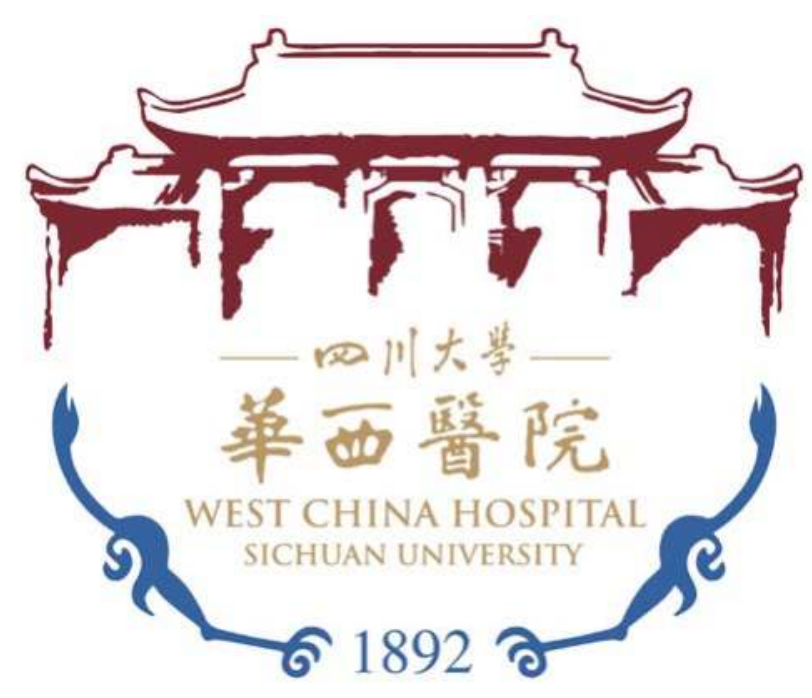

